\title{
Improved Delay-Dependent Stability Analysis for Neural Networks with Interval Time-Varying Delays
}

\author{
Jun-kang Tian and Yan-min Liu \\ School of Mathematics and Computer Science, Zunyi Normal College, Zunyi 563002, China \\ Correspondence should be addressed to Jun-kang Tian; zunytian@163.com
}

Received 20 October 2014; Accepted 27 November 2014

Academic Editor: P. Balasubramaniam

Copyright ( 2015 J.-k. Tian and Y.-m. Liu. This is an open access article distributed under the Creative Commons Attribution License, which permits unrestricted use, distribution, and reproduction in any medium, provided the original work is properly cited.

The problem of delay-dependent asymptotic stability analysis for neural networks with interval time-varying delays is considered based on the delay-partitioning method. Some less conservative stability criteria are established in terms of linear matrix inequalities (LMIs) by constructing a new Lyapunov-Krasovskii functional (LKF) in each subinterval and combining with reciprocally convex approach. Moreover, our criteria depend on both the upper and lower bounds on time-varying delay and its derivative, which is different from some existing ones. Finally, a numerical example is given to show the improved stability region of the proposed results.

\section{Introduction}

In the past decades, neural networks have been paid much attention due to their strong capability of information processing such as pattern recognition, image processing, fault diagnosis, and associative memories. Meanwhile, due to the finite switching speed of amplifiers, time-delay is inevitably encountered in real-world neural networks. Time-delays often cause instability and oscillation in neural networks. Therefore, many effects have been paid to delay-dependent stability analysis of neural networks with time-delays.

For the delay-dependent stability criteria of neural networks with time delays, the main purpose is to obtain a maximum value of the admissible delay such that the concerned systems are asymptotically stable. To enhance the feasible region of stability criteria, by using the free weighting matrix method, a new delay-dependent stability criterion for delayed neural networks was obtained in [1]. But some useful terms were ignored in [1] when estimating the upper bound on the derivative of the Lyapunov functionals. Then, the result was further improved in [2] by considering the relationships among some useful terms adequately. In order to reduce the conservatism, researchers have introduced the augmented Lyapunov functional method
[3] and the reciprocally convex approach [4]. Recently, the delay-partitioning approach which divides delay interval into some subintervals was employed to investigate the delaydependent stability problem of delayed neural networks [512]. In $[5,6]$, the delay-partitioning number was chosen as 2. In [5], some improved stability criteria were obtained by utilizing different free-weighting matrices in each delay subinterval for neural networks with time-varying delays. In [7-12], the delay-partitioning number was chosen as $m$, where $m \geqslant 1$. In [8], a weighting delay based method was introduced to derive the stability criteria for recurrent neural networks with time-varying delay. Different from some existing results, by employing weighting delays, the delay interval $[0, d(t)]$ was divided into some variable subintervals. In [10], complete delay-decomposing approach was introduced to derive asymptotic stability criterion for neural networks with time-varying delays by using reciprocally convex technique. Among those above methods, the delay-partitioning method is proven to be more effective than the free-weighting matrix approach and augmented Lyapunov functional method.

In the above mentioned results [1-12], the lower bound of time-varying delay for delayed neural networks is restricted to be 0 . However, in the real world, the time-varying delay may be an interval delay; that is, the lower bound of 
the delay is not restricted to be 0 . Therefore, many effects have been paid to delay-dependent stability analysis of neural networks with interval time-varying delay [13-17]. In [13], A piecewise delay method is introduced to derive several novel delay-dependent stability criteria for neural networks with interval time-varying delay. By using the delay decomposition method and a new convex combination technique, some new delay-dependent stability criteria were obtained in [14]. Recently, by constructing a new Lyapunov functional and dividing the lower bound of the time-varying delay, the asymptotic stability for cellular neural networks with interval time-varying delays is investigated in [15]. By dividing the lower and upper bounds of the time-varying delay and constructing an improved Lyapunov-Krasovskii functional, [16] obtained some delay-dependent stability criteria in terms of LMIs to reduce the conservatism. The proposed stability conditions are less conservative due to the novel delaypartitioning method and convex combination technique considered. By considering the sufficient information of neuron activation functions, [17] obtained some improved delay-dependent stability criteria for neural networks with interval time-varying delay. However, there are rooms for further improvement. Firstly, in [15], only the delay interval $\left[0, h_{1}\right]$ has been divided into $m$ segments. In this case, there is no connection between time-varying delay and each subinterval, which may lead to much conservatism. In [16], the delay intervals $\left[0, h_{1}\right]$ and $\left[h_{1}, h_{2}\right]$ have been divided into some segments and the information $\tau(t) \in\left[h_{1}, h_{2}\right]$ is considered, but the relation between the time-varying delay and each subinterval is not adequately considered, which may lead to considerable conservatism. Secondly, many stability conditions ignore the information of the lower bound of delay derivative. In fact, the lower bound of delay derivative plays an important role in improving stability region of the proposed results. Thirdly, the information of neuron activation functions is not adequately considered, which may lead to much conservatism.

In this paper, to show the merits of our new delaypartitioning method, we only need to divide the delay intervals $\left[0, h_{1}\right]$ and $\left[h_{1}, h_{2}\right]$ into 2 segments; that is, $\left[0, h_{1}\right]=$ $\left[0, h_{1} / 2\right] \cup\left[h_{1} / 2, h_{1}\right],\left[h_{1}, h_{2}\right]=\left[h_{1},\left(h_{1}+h_{2}\right) / 2\right] \cup\left[\left(h_{1}+\right.\right.$ $\left.h_{2}\right) / 2, h_{2}$ ], respectively. Some existing results [14-16] only consider the information $\tau(t) \in\left[h_{1}, h_{2}\right]$ and do not consider the information $\tau(t) \in\left[h_{1},\left(h_{1}+h_{2}\right) / 2\right]$ or $\tau(t) \in\left[\left(h_{1}+\right.\right.$ $\left.h_{2}\right) / 2, h_{2}$ ], which may lead to considerable conservatism. Thus, when dealing with the time derivative of $V\left(z_{t}\right)$, not only the information $\tau(t) \in\left[h_{1}, h_{2}\right]$ but also the information $\tau(t) \epsilon$ $\left[h_{1},\left(h_{1}+h_{2}\right) / 2\right]$ and $\tau(t) \in\left[\left(h_{1}+h_{2}\right) / 2, h_{2}\right]$ is considered in our paper. So the information between time-varying delay $\tau(t)$ and each subinterval is considered adequately, which may play an important role in reducing conservatism of derived results. By taking more information of the lower bound of delay derivative, an augmented LKF is introduced to derive two stability criteria for neural networks with interval time-varying delay. Then, by employing reciprocally convex approach, some less conservative delay-dependent stability criteria are presented in terms of LMI. Finally, a numerical example is given to show the effectiveness of the proposed method.

\section{Problem Formulation}

Consider the following neural networks with interval timevarying delay:

$$
\dot{x}(t)=-C x(t)+A g(x(t))+B g(x(t-\tau(t)))+\mu,
$$

where $x(t)=\left[x_{1}(t), x_{2}(t), \ldots, x_{n}(t)\right]^{T} \in \mathscr{R}^{n}$ is the neuron state vector, $g(x(\cdot))=\left[g_{1}\left(x_{1}(\cdot)\right), g_{2}\left(x_{2}(\cdot)\right), \ldots, g_{n}\left(x_{n}(\cdot)\right)\right]^{T} \in$ $\mathscr{R}^{n}$ denotes the neuron activation function, and $\mu=$ $\left(\mu_{1}, \mu_{2}, \ldots, \mu_{n}\right)^{T} \in \mathscr{R}^{n}$ is a constant input vector. $A \in \mathscr{R}^{n \times n}$ is the connection weight matrix and $B \in \mathscr{R}^{n \times n}$ is the delayed connection weight matrix. $C=\operatorname{diag}\left(C_{1}, C_{2}, \ldots, C_{n}\right)$ is a diagonal matrix with $C_{i}>0, i=1,2, \ldots, n . \tau(t)$ is timevarying continuous function that satisfies $0 \leqslant h_{1} \leqslant \tau(t) \leqslant h_{2}$, $u_{1} \leqslant \dot{\tau}(t) \leqslant u_{2}$, where $h_{1}, h_{2}$ and $u_{1}, u_{2}$ are constants. In addition, it is assumed that each neuron activation function $g_{i}(\cdot), i=1,2, \ldots, n$, satisfies the following condition:

$$
k_{i}^{-} \leqslant \frac{g_{i}(x)-g_{i}(y)}{x-y} \leqslant k_{i}^{+}, \quad \forall x, y \in R, x \neq y, i=1,2, \ldots, n,
$$

where $k_{i}^{-}, k_{i}^{+}, i=1,2, \ldots, n$ are constants.

Assuming that $x^{*}=\left[x_{1}^{*}, x_{2}^{*}, \ldots, x_{n}^{*}\right]^{T}$ is the equilibrium point of (1). By the transformation $z(\cdot)=x(\cdot)-x^{*},(1)$ can be converted to the following form:

$$
\dot{z}(t)=-C z(t)+A f(z(t))+B f(z(t-\tau(t))),
$$

where $z(t)=\left[z_{1}(t), z_{2}(t), \ldots, z_{n}(t)\right]^{T}, f(z(\cdot))=\left[f_{1}\left(z_{1}(\cdot)\right)\right.$, $\left.f_{2}\left(z_{2}(\cdot)\right), \ldots, f_{n}\left(z_{n}(\cdot)\right)\right]^{T}$, and $f_{i}\left(z_{i}(\cdot)\right)=g_{i}\left(z_{i}(\cdot)+x_{i}^{*}\right)-g_{i}\left(x_{i}^{*}\right)$, $i=1,2, \ldots, n$. According to inequality (2), we obtain

$$
k_{i}^{-} \leqslant \frac{f_{i}\left(z_{i}(t)\right)}{z_{i}(t)} \leqslant k_{i}^{+} \quad f_{i}(0)=0, i=1,2, \ldots, n .
$$

Lemma 1 (see [18]). For any constant matrix $Z \in \mathscr{R}^{n \times n}, Z=$ $Z^{T}>0$, scalars $h_{2}>h_{1}>0$; then

$$
\begin{aligned}
& -\left(h_{2}-h_{1}\right) \int_{t-h_{2}}^{t-h_{1}} x^{T}(s) Z x(s) \mathrm{d} s \\
& \leqslant-\int_{t-h_{2}}^{t-h_{1}} x^{T}(s) d s Z \int_{t-h_{2}}^{t-h_{1}} x(s) \mathrm{d} s .
\end{aligned}
$$

Lemma 2 (see [19]). Let $f_{1}, f_{2}, \ldots, f_{N}: \mathscr{R}^{m} \rightarrow \mathscr{R}$ have positive values in an open subset $D$ of $\mathscr{R}^{m}$. Then, the reciprocally convex combination of $f_{i}$ over D satisfies

$$
\min _{\left\{\alpha_{i} \mid \alpha_{i}>0, \sum_{i} \alpha_{i}=1\right\}} \sum_{i} \frac{1}{\alpha_{i}} f_{i}(t)=\sum_{i} f_{i}(t)+\max _{g_{i, j}(t)} \sum_{i \neq j} g_{i, j}(t)
$$

subject to

$$
\left\{g_{i, j}: R^{m} \longrightarrow R, g_{j, i}(t) \triangleq g_{i, j}(t),\left[\begin{array}{cc}
f_{i}(t) & g_{i, j}(t) \\
g_{i, j}(t) & f_{j}(t)
\end{array}\right] \geqslant 0\right\} .
$$




\section{Main Results}

Theorem 3. For given positive scalars $h_{1}, h_{2}, h=h_{2}-$ $h_{1}$, and any scalars $u_{1}, u_{2}$, diagonal matrices $K_{1}=$ $\operatorname{diag}\left(k_{1}^{-}, k_{2}^{-}, \ldots, k_{n}^{-}\right), K_{2}=\operatorname{diag}\left(k_{1}^{+}, k_{2}^{+}, \ldots, k_{n}^{+}\right)$, and system (3) is globally asymptotically stable for $0 \leqslant h_{1} \leqslant \tau(t) \leqslant h_{2}$, $u_{1} \leqslant \dot{\tau}(t) \leqslant u_{2}$ if there exist symmetric positive matrices $P, X=$ $\left[X_{i j}\right]_{2 \times 2}, Q=\left[Q_{i j}\right]_{2 \times 2}, \bar{P}=\left[P_{i j}\right]_{2 \times 2}, R_{i}(i=1,2,3,4)$, positive

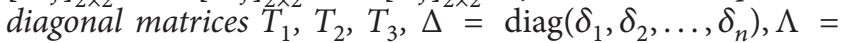
$\operatorname{diag}\left(\lambda_{1}, \lambda_{2}, \ldots, \lambda_{n}\right)$, and any matrices $S_{1}, S_{2}$ with appropriate dimensions, satisfying the following LMIs:

$$
\begin{gathered}
{\left[\begin{array}{cc}
\Sigma+\Phi+\Pi_{1} & \mathscr{A}^{T} R \\
* & -R
\end{array}\right]<0,} \\
{\left[\begin{array}{cc}
\Sigma+\Phi+\Pi_{2} & \mathscr{A}^{T} R \\
* & -R
\end{array}\right]<0,} \\
{\left[\begin{array}{cc}
R_{1} & S_{1} \\
* & R_{1}
\end{array}\right]>0} \\
{\left[\begin{array}{cc}
R_{2} & S_{2} \\
* & R_{2}
\end{array}\right]>0}
\end{gathered}
$$

where

$$
\begin{aligned}
& \Sigma=\left[\begin{array}{ccccccccc}
\Sigma_{11} & X_{12} & 0 & 0 & 0 & 0 & 0 & Q_{12}-P_{12} & -Y_{12} \\
* & X_{22}-X_{11} & -X_{12} & 0 & 0 & 0 & 0 & 0 & 0 \\
* & * & -X_{22}-Y & 0 & 0 & 0 & 0 & 0 & 0 \\
* & * & * & \Sigma_{44} & 0 & 0 & -\left(1-u_{2}\right) Q_{12} & 0 & 0 \\
* & * & * & * & \Sigma_{55} & \Sigma_{56} & \Sigma_{57} & 0 & Y_{12} \\
* & * & * & * & * & \Sigma_{66} & (\Lambda-\Delta) B & 0 & 0 \\
* & * & * & * & * & * & -\left(1-u_{2}\right) Q_{22} & 0 & 0 \\
* & * & * & * & * & * & * & Q_{22}-P_{22} & 0 \\
* & * & * & * & * & * & * & * & Y_{22}-Y_{11}
\end{array}\right] \\
& \Phi=\left[\begin{array}{ccccccccc}
-2 K_{2} T_{3} K_{1} & 0 & 0 & 0 & 0 & 0 & 0 & T_{3}\left(K_{1}+K_{2}\right) & 0 \\
* & 0 & 0 & 0 & 0 & 0 & 0 & 0 & 0 \\
* & * & 0 & 0 & 0 & 0 & 0 & 0 & 0 \\
* & * & * & -2 K_{2} T_{2} K_{1} & 0 & 0 & T_{2}\left(K_{1}+K_{2}\right) & 0 & 0 \\
* & * & * & * & -2 K_{2} T_{1} K_{1} & T_{1}\left(K_{1}+K_{2}\right) & 0 & 0 & 0 \\
* & * & * & * & * & -2 T_{1} & 0 & 0 & 0 \\
* & * & * & * & * & * & -2 T_{2} & 0 & 0 \\
* & * & * & * & * & * & * & -2 T_{3} & 0 \\
* & * & * & * & * & * & * & * & 0
\end{array}\right], \\
& \Pi_{1}=\left[\begin{array}{ccccccccc}
-R_{1}-R_{4} & S_{1} & 0 & R_{1}-S_{1} & 0 & 0 & 0 & 0 & R_{4} \\
* & -R_{1}-R_{2} & R_{2} & R_{1}-S_{1}^{T} & 0 & 0 & 0 & 0 & 0 \\
* & * & -R_{2} & 0 & 0 & 0 & 0 & 0 & 0 \\
* & * & * & -2 R_{1}+S_{1}+S_{1}^{T} & 0 & 0 & 0 & 0 & 0 \\
* & * & * & * & -R_{3} & 0 & 0 & 0 & R_{3} \\
* & * & * & * & * & 0 & 0 & 0 & 0 \\
* & * & * & * & * & * & 0 & 0 & 0 \\
* & * & * & * & * & * & * & 0 & 0 \\
* & * & * & * & * & * & * & * & -R_{3}-R_{4}
\end{array}\right], \\
& \Pi_{2}=\left[\begin{array}{ccccccccc}
-R_{1}-R_{4} & R_{1} & 0 & 0 & 0 & 0 & 0 & 0 & R_{4} \\
* & -R_{1}-R_{2} & S_{2} & R_{2}-S_{2} & 0 & 0 & 0 & 0 & 0 \\
* & * & -R_{2} & R_{2}-S_{2}^{T} & 0 & 0 & 0 & 0 & 0 \\
* & * & * & -2 R_{2}+S_{2}+S_{2}^{T} & 0 & 0 & 0 & 0 & 0 \\
* & * & * & * & -R_{3} & 0 & 0 & 0 & R_{3} \\
* & * & * & * & * & 0 & 0 & 0 & 0 \\
* & * & * & * & * & * & 0 & 0 & 0 \\
* & * & * & * & * & * & * & 0 & 0 \\
* & * & * & * & * & * & * & * & -R_{3}-R_{4}
\end{array}\right],
\end{aligned}
$$




$$
\begin{aligned}
& \Sigma_{11}=X_{11}+Q_{11}-P_{11}-Y_{22}, \\
& \Sigma_{44}=-\left(1-u_{2}\right) Q_{11}+\left(1-u_{1}\right) Y \text {, } \\
& \Sigma_{55}=-P C-C^{T} P-\left(K_{2} \Delta-K_{1} \Lambda\right) C \\
& -C^{T}\left(K_{2} \Delta-K_{1} \Lambda\right)+P_{11}+Y_{11}, \\
& \Sigma_{56}=P A-C(\Lambda-\Delta)+\left(K_{2} \Delta-K_{1} \Lambda\right) A+P_{12} \text {, } \\
& \Sigma_{57}=P B+\left(K_{2} \Delta-K_{1} \Lambda\right) B, \\
& \Sigma_{66}=(\Lambda-\Delta) A+A^{T}(\Lambda-\Delta)+P_{22}, \\
& \mathscr{A}=\left[\begin{array}{lllllllll}
0 & 0 & 0 & 0 & -C & A & B & 0 & 0
\end{array}\right], \\
& R=\frac{h^{2}}{4}\left(R_{1}+R_{2}\right)+\frac{h_{1}^{2}}{4}\left(R_{3}+R_{4}\right) \text {. }
\end{aligned}
$$

Proof. We construct a new LKF as

$$
V\left(z_{t}\right)=\sum_{i=1}^{5} V_{i}\left(z_{t}\right),
$$

where

$$
\begin{aligned}
& V_{1}\left(z_{t}\right)=\int_{t-h_{1}-(h / 2)}^{t-h_{1}}\left[z\left(s-\frac{h}{2}\right)\right]^{T}\left[\begin{array}{cc}
X_{11} & X_{12} \\
* & X_{22}
\end{array}\right] \\
& {\left[\begin{array}{c}
z(s) \\
z\left(s-\frac{h}{2}\right)
\end{array}\right] \mathrm{d} s} \\
& +\int_{t-\left(h_{1} / 2\right)}^{t}\left[z\left(s-\frac{h_{1}}{2}\right)\right]^{T}\left[\begin{array}{cc}
Y_{11} & Y_{12} \\
* & Y_{22}
\end{array}\right] \\
& {\left[\begin{array}{c}
z(s) \\
z\left(s-\frac{h_{1}}{2}\right)
\end{array}\right] \mathrm{d} s} \\
& V_{2}\left(z_{t}\right)=z^{T}(t) P z(t)+2 \sum_{i=1}^{n}\left\{\int_{0}^{z_{i}(t)} \lambda_{i}\left(f_{i}(s)-k_{i}^{-} s\right) \mathrm{d} s\right. \\
& \left.+\int_{0}^{z_{i}(t)} \delta_{i}\left(k_{i}^{+} s-f_{i}(s)\right) \mathrm{d} s\right\}, \\
& V_{3}\left(z_{t}\right)=\int_{t-\tau(t)}^{t-h_{1}}\left[\begin{array}{c}
z(s) \\
f(z(s))
\end{array}\right]^{T}\left[\begin{array}{cc}
Q_{11} & Q_{12} \\
* & Q_{22}
\end{array}\right]\left[\begin{array}{c}
z(s) \\
f(z(s))
\end{array}\right] \mathrm{d} s \\
& +\int_{t-h_{1}}^{t}\left[\begin{array}{c}
z(s) \\
f(z(s))
\end{array}\right]^{T}\left[\begin{array}{cc}
P_{11} & P_{12} \\
* & P_{22}
\end{array}\right]\left[\begin{array}{c}
z(s) \\
f(z(s))
\end{array}\right] \mathrm{d} s, \\
& V_{4}\left(z_{t}\right)=\int_{t-h_{2}}^{t-\tau(t)} z^{T}(s) Y z(s) \mathrm{d} s,
\end{aligned}
$$

$$
\begin{aligned}
V_{5}\left(z_{t}\right)= & \frac{h}{2} \int_{-\left(h_{1}+h_{2}\right) / 2}^{-h_{1}} \int_{t+\theta}^{t} \dot{z}^{T}(s) R_{1} \dot{z}(s) \mathrm{d} s \mathrm{~d} \theta \\
& +\frac{h}{2} \int_{-h_{2}}^{-\left(h_{1}+h_{2}\right) / 2} \int_{t+\theta}^{t} \dot{z}^{T}(s) R_{2} \dot{z}(s) \mathrm{d} s \mathrm{~d} \theta \\
& +\frac{h_{1}}{2} \int_{-\left(h_{1} / 2\right)}^{0} \int_{t+\theta}^{t} \dot{z}^{T}(s) R_{3} \dot{z}(s) \mathrm{d} s \mathrm{~d} \theta \\
& +\frac{h_{1}}{2} \int_{-h_{1}}^{-\left(h_{1} / 2\right)} \int_{t+\theta}^{t} \dot{z}^{T}(s) R_{4} \dot{z}(s) \mathrm{d} s \mathrm{~d} \theta
\end{aligned}
$$

Remark 4 . When constructing the $V_{1}\left(z_{t}\right)$, we not only divide the delay interval $\left[0, h_{1}\right]$ but also divide the delay interval $\left[h_{1}, h_{2}\right]$ into 2 segments; that is, $\left[0, h_{1}\right]=\left[0, h_{1} / 2\right] \cup\left[h_{1} / 2, h_{1}\right]$, $\left[h_{1}, h_{2}\right]=\left[h_{1},\left(h_{1}+h_{2}\right) / 2\right] \cup\left[\left(h_{1}+h_{2}\right) / 2, h_{2}\right]$, respectively, which may play an important role in reducing conservatism of derived results.

Remark 5. Since $V_{4}\left(z_{t}\right)$ considers the information of the lower bound of delay derivative, the LKF in this paper is more general than that in [14-16]. So the obtained stability criteria may be less conservative than the existing ones.

The time derivative of $V\left(z_{t}\right)$ along the trajectories of system (3) yields

$$
\dot{V}\left(z_{t}\right)=\sum_{i=1}^{5} \dot{V}_{i}\left(z_{t}\right),
$$

where

$$
\begin{aligned}
\dot{V}_{1}\left(z_{t}\right)= & {\left[\begin{array}{c}
z\left(t-h_{1}\right) \\
z\left(t-\frac{h_{1}+h_{2}}{2}\right)
\end{array}\right]^{T}\left[\begin{array}{cc}
X_{11} & X_{12} \\
* & X_{22}
\end{array}\right] } \\
& \cdot\left[\begin{array}{c}
z\left(t-h_{1}\right) \\
z\left(t-\frac{h_{1}+h_{2}}{2}\right)
\end{array}\right]
\end{aligned}
$$




$$
\begin{aligned}
& -\left[\begin{array}{c}
z\left(t-\frac{h_{1}+h_{2}}{2}\right) \\
z\left(t-h_{2}\right)
\end{array}\right]^{T}\left[\begin{array}{cc}
X_{11} & X_{12} \\
* & X_{22}
\end{array}\right] \\
& {\left[\begin{array}{c}
z\left(t-\frac{h_{1}+h_{2}}{2}\right) \\
z\left(t-h_{2}\right)
\end{array}\right]} \\
& -\left[\begin{array}{c}
z(t) \\
z\left(t-\frac{h_{1}}{2}\right)
\end{array}\right]^{T}\left[\begin{array}{cc}
Y_{11} & Y_{12} \\
* & Y_{22}
\end{array}\right]\left[\begin{array}{c}
z(t) \\
z\left(t-\frac{h_{1}}{2}\right)
\end{array}\right] \\
& -\left[\begin{array}{c}
z\left(t-\frac{h_{1}}{2}\right) \\
z\left(t-h_{1}\right)
\end{array}\right]^{T}\left[\begin{array}{cc}
Y_{11} & Y_{12} \\
* & Y_{22}
\end{array}\right]\left[\begin{array}{c}
z\left(t-\frac{h_{1}}{2}\right) \\
z\left(t-h_{1}\right)
\end{array}\right], \\
& \dot{V}_{2}\left(z_{t}\right)=2 z^{T}(t) P \dot{z}(t)+2\left[f(z(t))-K_{1} z(t)\right]^{T} \Lambda \dot{z}(t) \\
& +2\left[K_{2} z(t)-f(z(t))\right]^{T} \Delta \dot{z}(t) \\
& =2 z^{T}(t) P[-C z(t)+A f(z(t))+B f(z(t-\tau(t)))] \\
& +2\left[f(z(t))-K_{1} z(t)\right]^{T} \Lambda[-C z(t)+A f(z(t)) \\
& +B f(z(t-\tau(t)))] \\
& +2\left[K_{2} z(t)-f(z(t))\right]^{T} \Delta[-C z(t)+A f(z(t)) \\
& +B f(z(t-\tau(t)))], \\
& \dot{V}_{3}\left(z_{t}\right) \leqslant\left[\begin{array}{c}
z\left(t-h_{1}\right) \\
f\left(z\left(t-h_{1}\right)\right)
\end{array}\right]^{T}\left[\begin{array}{cc}
Q_{11} & Q_{12} \\
* & Q_{22}
\end{array}\right]\left[\begin{array}{c}
z\left(t-h_{1}\right) \\
f\left(z\left(t-h_{1}\right)\right)
\end{array}\right] \\
& -\left(1-u_{2}\right)\left[\begin{array}{c}
z(t-\tau(t)) \\
f(z(t-\tau(t)))
\end{array}\right]^{T} \\
& \cdot\left[\begin{array}{cc}
Q_{11} & Q_{12} \\
* & Q_{22}
\end{array}\right]\left[\begin{array}{c}
z(t-\tau(t)) \\
f(z(t-\tau(t)))
\end{array}\right] \\
& +\left[\begin{array}{c}
z(t) \\
f(z(t))
\end{array}\right]^{T}\left[\begin{array}{cc}
P_{11} & P_{12} \\
* & P_{22}
\end{array}\right]\left[\begin{array}{c}
z(t) \\
f(z(t))
\end{array}\right] \\
& -\left[\begin{array}{c}
z\left(t-h_{1}\right) \\
f\left(z\left(t-h_{1}\right)\right)
\end{array}\right]^{T}\left[\begin{array}{cc}
P_{11} & P_{12} \\
* & P_{22}
\end{array}\right]\left[\begin{array}{c}
z\left(t-h_{1}\right) \\
f\left(z\left(t-h_{1}\right)\right)
\end{array}\right], \\
& \dot{V}_{4}\left(z_{t}\right) \leqslant\left(1-u_{1}\right) z^{T}(t-\tau(t)) Y z(t-\tau(t)) \\
& -z^{T}\left(t-h_{2}\right) Y z\left(t-h_{2}\right) .
\end{aligned}
$$

By (16), we obtain

$$
\dot{V}_{1}\left(z_{t}\right)+\dot{V}_{2}\left(z_{t}\right)+\dot{V}_{3}\left(z_{t}\right)+\dot{V}_{4}\left(z_{t}\right) \leqslant \zeta^{T}(t) \Sigma \zeta(t),
$$

where $\Sigma$ is defined in (8). Consider the following:

$$
\begin{aligned}
& \zeta^{T}(t)=[ z^{T}\left(t-h_{1}\right) z^{T}\left(t-\frac{h_{1}+h_{2}}{2}\right) \\
& \cdot z^{T}\left(t-h_{2}\right) z^{T}(t-\tau(t)) \\
& \cdot z^{T}(t) f^{T}(z(t)) f^{T}(z(t-\tau(t))) \\
&\left.\cdot f^{T}\left(z\left(t-h_{1}\right)\right) f^{T}\left(z\left(t-\frac{h_{1}}{2}\right)\right)\right], \\
& \dot{V}_{5}\left(z_{t}\right)=\dot{z}^{T}(t)\left[\frac{h^{2}}{2}\left(R_{1}+R_{2}\right)+\frac{h_{1}^{2}}{2}\left(R_{3}+R_{4}\right)\right] \dot{z}(t) \\
& \quad-\frac{h}{2} \int_{t-\left(h_{1}+h_{2}\right) / 2}^{t-h_{1}} \dot{z}^{T}(s) R_{1} \dot{z}(s) \mathrm{d} s \\
&-\frac{h}{2} \int_{t-h_{2}}^{t-\left(\left(h_{1}+h_{2}\right) / 2\right)} \dot{z}^{T}(s) R_{2} \dot{z}(s) \mathrm{d} s \\
&-\frac{h_{1}}{2} \int_{t-\left(h_{1} / 2\right)}^{t} \dot{z}^{T}(s) R_{3} \dot{z}(s) \mathrm{d} s \\
& \quad-\frac{h_{1}}{2} \int_{t-h_{1}}^{t-\left(h_{1} / 2\right)} \dot{z}^{T}(s) R_{4} \dot{z}(s) \mathrm{d} s .
\end{aligned}
$$

Furthermore, there exist positive diagonal matrices $T_{1}, T_{2}, T_{3}$, such that the following inequalities hold based on (4):

$$
\begin{aligned}
0 \leqslant & -2 f^{T}(z(t)) T_{1} f(z(t))+2 z^{T}(t) T_{1}\left(K_{1}+K_{2}\right) f(z(t)) \\
& -2 z^{T}(t) K_{2} T_{1} K_{1} z(t) \\
& -2 f^{T}(z(t-\tau(t))) T_{2} f(z(t-\tau(t))) \\
& +2 z^{T}(t-\tau(t)) T_{2}\left(K_{1}+K_{2}\right) f(z(t-\tau(t))) \\
& -2 z^{T}(t-\tau(t)) K_{2} T_{2} K_{1} z(t-\tau(t)) \\
& -2 f^{T}\left(z\left(t-h_{1}\right)\right) T_{3} f\left(z\left(t-h_{1}\right)\right) \\
& +2 z^{T}\left(t-h_{1}\right) T_{3}\left(K_{1}+K_{2}\right) f\left(z\left(t-h_{1}\right)\right) \\
& -2 z^{T}\left(t-h_{1}\right) K_{2} T_{3} K_{1} z\left(t-h_{1}\right) \\
= & \zeta^{T}(t) \Phi \zeta(t) .
\end{aligned}
$$


For $h_{1} \leqslant \tau(t) \leqslant\left(h_{1}+h_{2}\right) / 2$, by use of Lemma 1 , we obtain

$$
\begin{gathered}
-\frac{h}{2} \int_{t-\left(\left(h_{1}+h_{2}\right) / 2\right)}^{t-h_{1}} \dot{z}^{T}(s) R_{1} \dot{z}(s) \mathrm{d} s \\
=-\frac{h}{2} \int_{t-\left(\left(h_{1}+h_{2}\right) / 2\right)}^{t-\tau(t)} \dot{z}^{T}(s) R_{1} \dot{z}(s) \mathrm{d} s \\
-\frac{h}{2} \int_{t-\tau(t)}^{t-h_{1}} \dot{z}^{T}(s) R_{1} \dot{z}(s) \mathrm{d} s .
\end{gathered}
$$

Let

$$
\begin{aligned}
& e_{1}(t)=\int_{t-\left(\left(h_{1}+h_{2}\right) / 2\right)}^{t-\tau(t)} \dot{z}(s) \mathrm{d} s, \\
& e_{2}(t)=\int_{t-\tau(t)}^{t-h_{1}} \dot{z}(s) \mathrm{d} s
\end{aligned}
$$

then

$$
\begin{aligned}
& -\frac{h}{2} \int_{t-\left(\left(h_{1}+h_{2}\right) / 2\right)}^{t-h_{1}} \dot{z}^{T}(s) R_{1} \dot{z}(s) \mathrm{d} s \\
& \leqslant-\frac{h / 2}{\left(\left(h_{1}+h_{2}\right) / 2\right)-\tau(t)} e_{1}^{T}(t) R_{1} e_{1}(t) \\
& \quad-\frac{h / 2}{\tau(t)-h_{1}} e_{2}^{T}(t) R_{1} e_{2}(t) \\
& =-e_{1}^{T}(t) R_{1} e_{1}(t)-\frac{\tau(t)-h_{1}}{\left(\left(h_{1}+h_{2}\right) / 2\right)-\tau(t)} e_{1}^{T}(t) R_{1} e_{1}(t) \\
& \quad-e_{2}^{T} R_{1} e_{2}(t)-\frac{\left(\left(h_{1}+h_{2}\right) / 2\right)-\tau(t)}{\tau(t)-h_{1}} e_{2}^{T}(t) R_{1} e_{2}(t) .
\end{aligned}
$$

If $\left[\begin{array}{cc}R_{1} & S_{1} \\ * & R_{1}\end{array}\right]>0$, then, by using Lemma 2 , we obtain

$$
\begin{gathered}
{\left[\sqrt{\frac{\tau(t)-h_{1}}{\left(\left(h_{1}+h_{2}\right) / 2\right)-\tau(t)}} e_{1}(t)\right.} \\
\left.-\sqrt{\frac{\left(\left(h_{1}+h_{2}\right) / 2\right)-\tau(t)}{\tau(t)-h_{1}}} e_{2}(t)\right]^{T}\left[\begin{array}{ll}
R_{1} & S_{1} \\
* & R_{1}
\end{array}\right] \\
\cdot\left[\begin{array}{l}
\sqrt{\frac{\tau(t)-h_{1}}{\left(\left(h_{1}+h_{2}\right) / 2\right)-\tau(t)}} e_{1}(t) \\
-\sqrt{\frac{\left(\left(h_{1}+h_{2}\right) / 2\right)-\tau(t)}{\tau(t)-h_{1}}} e_{2}(t)
\end{array}\right] \geqslant 0,
\end{gathered}
$$

which implies

$$
\begin{gathered}
-\frac{\tau(t)-h_{1}}{\left(\left(h_{1}+h_{2}\right) / 2\right)-\tau(t)} e_{1}^{T}(t) R_{1} e_{1}(t) \\
-\frac{\left(\left(h_{1}+h_{2}\right) / 2\right)-\tau(t)}{\tau(t)-h_{1}} e_{2}^{T}(t) R_{1} e_{2}(t) \\
\quad \leqslant-e_{1}^{T}(t) S_{1} e_{2}(t)-e_{2}^{T}(t) S_{1}^{T} e_{1}(t) .
\end{gathered}
$$

Then, from (19)-(24), we obtain

$$
\begin{aligned}
-\frac{h}{2} \int_{t-\left(\left(h_{1}+h_{2}\right) / 2\right)}^{t-h_{1}} \dot{z}^{T}(s) R_{1} \dot{z}(s) \mathrm{d} s \\
\leqslant-e_{1}^{T}(t) R_{1} e_{1}(t)-e_{2}^{T}(t) R_{1} e_{2}(t) \\
-e_{1}^{T}(t) S_{1} e_{2}(t)-e_{2}^{T}(t) S_{1}^{T} e_{1}(t) \\
=-\left[z(t-\tau(t))-z\left(t-\frac{h_{1}+h_{2}}{2}\right)\right]^{T} \\
\quad \cdot R_{1}\left[z(t-\tau(t))-z\left(t-\frac{h_{1}+h_{2}}{2}\right)\right] \\
-\left[z\left(t-h_{1}\right)-z(t-\tau(t))\right]^{T} \\
\cdot R_{1}\left[z\left(t-h_{1}\right)-z(t-\tau(t))\right] \\
-\left[z(t-\tau(t))-z\left(t-\frac{h_{1}+h_{2}}{2}\right)\right]^{T} \\
\cdot S_{1}\left[z\left(t-h_{1}\right)-z(t-\tau(t))\right] \\
-\left[z\left(t-h_{1}\right)-z(t-\tau(t))\right]^{T} \\
\cdot S_{1}^{T}\left[z(t-\tau(t))-z\left(t-\frac{h_{1}+h_{2}}{2}\right)\right],
\end{aligned}
$$

$-\frac{h}{2} \int_{t-h_{2}}^{t-\left(\left(h_{1}+h_{2}\right) / 2\right)} \dot{z}^{T}(s) R_{2} \dot{z}(s) \mathrm{d} s$

$$
\leqslant-\left[z\left(t-\frac{h_{1}+h_{2}}{2}\right)-z\left(t-h_{2}\right)\right]^{T}
$$

$$
\cdot R_{2}\left[z\left(t-\frac{h_{1}+h_{2}}{2}\right)-z\left(t-h_{2}\right)\right] \text {, }
$$

$-\frac{h_{1}}{2} \int_{t-\left(h_{1} / 2\right)}^{t} \dot{z}^{T}(s) R_{3} \dot{z}(s) \mathrm{d} s$

$$
\begin{aligned}
& -\frac{h_{1}}{2} \int_{t-h_{1}}^{t-\left(h_{1} / 2\right)} \dot{z}^{T}(s) R_{4} \dot{z}(s) \mathrm{d} s \\
\leqslant & {\left[z(t)-z\left(t-\frac{h_{1}}{2}\right)\right]^{T} R_{3}\left[z(t)-z\left(t-\frac{h_{1}}{2}\right)\right] } \\
& -\left[z\left(t-\frac{h_{1}}{2}\right)-z\left(t-h_{1}\right)\right]^{T} \\
& \cdot R_{4}\left[z\left(t-\frac{h_{1}}{2}\right)-z\left(t-h_{1}\right)\right] .
\end{aligned}
$$

Therefore

$$
\dot{V}_{5}\left(z_{t}\right) \leqslant \zeta^{T}(t)\left(\Pi_{1}+\mathscr{A}^{T} R \mathscr{A}\right) \zeta(t),
$$

where $\Pi_{1}, \mathscr{A}, R$ are defined in (8).

From (16)-(28), we obtain

$$
\dot{V}\left(z_{t}\right) \leqslant \zeta^{T}(t)\left(\Sigma+\Phi+\Pi_{1}+\mathscr{A}^{T} R \mathscr{A}\right) \zeta(t) .
$$


If $\Sigma+\Phi+\Pi_{1}+\mathscr{A}^{T} R \mathscr{A}<0$, then there exists a scalar $\varepsilon>0$, such that

$$
\dot{V}\left(z_{t}\right) \leqslant-\varepsilon \zeta^{T}(t) \zeta(t) \leqslant-\varepsilon z^{T}(t) z(t)<0, \quad \forall z(t) \neq 0 .
$$

Thus, according to [20], system (3) is globally asymptotically stable for $h_{1} \leqslant \tau(t) \leqslant\left(h_{1}+h_{2}\right) / 2$. By Schur complement, $\Sigma+\Phi+\Pi_{1}+\mathscr{A}^{T} R \mathscr{A}<0$ is equivalent to (8).

For $\left(h_{1}+h_{2}\right) / 2 \leqslant \tau(t) \leqslant h_{2}$, similar to $h_{1} \leqslant \tau(t) \leqslant\left(h_{1}+\right.$ $\left.h_{2}\right) / 2$, we obtain

$$
\begin{aligned}
-\frac{h}{2} & \int_{t-\left(\left(h_{1}+h_{2}\right) / 2\right)}^{t-h_{1}} \dot{z}^{T}(s) R_{1} \dot{z}(s) \mathrm{ds} \\
\leqslant & -\left[z\left(t-h_{1}\right)-z\left(t-\frac{h_{1}+h_{2}}{2}\right)\right]^{T} \\
& \cdot R_{1}\left[z\left(t-h_{1}\right)-z\left(t-\frac{h_{1}+h_{2}}{2}\right)\right] .
\end{aligned}
$$

If $\left[\begin{array}{cc}R_{2} & S_{2} \\ * & R_{2}\end{array}\right]>0$, then we obtain

$$
\begin{aligned}
-\frac{h}{2} & \int_{t-h_{2}}^{t-\left(\left(h_{1}+h_{2}\right) / 2\right)} \dot{z}^{T}(s) R_{2} \dot{z}(s) \mathrm{d} s \\
\leqslant & -e_{3}^{T}(t) R_{2} e_{3}(t)-e_{4}^{T}(t) R_{2} e_{4}(t) \\
& -e_{3}^{T}(t) S_{2} e_{4}(t)-e_{4}^{T}(t) S_{2}^{T} e_{3}(t) \\
= & -\left[z\left(t-\frac{h_{1}+h_{2}}{2}\right)-z(t-\tau(t))\right]^{T} \\
& \cdot R_{2}\left[z\left(t-\frac{h_{1}+h_{2}}{2}\right)-z(t-\tau(t))\right] \\
& -\left[z(t-\tau(t))-z\left(t-h_{2}\right)\right]^{T} \\
& \cdot R_{2}\left[z(t-\tau(t))-z\left(t-h_{2}\right)\right] \\
& -\left[z\left(t-\frac{h_{1}+h_{2}}{2}\right)-z(t-\tau(t))\right]^{T} \\
& \cdot S_{2}\left[z(t-\tau(t))-z\left(t-h_{2}\right)\right] \\
& -\left[z(t-\tau(t))-z\left(t-h_{2}\right)\right]^{T} \\
& \cdot S_{2}^{T}\left[z\left(t-\frac{h_{1}+h_{2}}{2}\right)-z(t-\tau(t))\right],
\end{aligned}
$$

where

$$
e_{3}(t)=\int_{t-\tau(t)}^{t-\left(\left(h_{1}+h_{2}\right) / 2\right)} \dot{z}(s) \mathrm{d} s, \quad e_{4}(t)=\int_{t-h_{2}}^{t-\tau(t)} \dot{z}(s) \mathrm{d} s .
$$

Therefore, by (27), (31), and (32), we obtain

$$
\dot{V}_{5}\left(z_{t}\right) \leqslant \zeta^{T}(t)\left(\Pi_{2}+\mathscr{A}^{T} R \mathscr{A}\right) \zeta(t),
$$

where $\Pi_{2}, \mathscr{A}, R$ are defined in (9).
From (16)-(19) and (34) we obtain

$$
\dot{V}\left(z_{t}\right) \leqslant \zeta^{T}(t)\left(\Sigma+\Phi+\Pi_{2}+\mathscr{A}^{T} R \mathscr{A}\right) \zeta(t) .
$$

If $\Sigma+\Phi+\Pi_{2}+\mathscr{A}^{T} R \mathscr{A}<0$, then there exists a scalar $\varepsilon_{1}>0$, such that

$$
\dot{V}\left(z_{t}\right) \leqslant-\varepsilon_{1} \zeta^{T}(t) \zeta(t) \leqslant-\varepsilon_{1} z^{T}(t) z(t)<0, \quad \forall z(t) \neq 0 .
$$

Thus, according to [20], system (3) is globally asymptotically stable for $\left(h_{1}+h_{2}\right) / 2 \leqslant \tau(t) \leqslant h_{2}$. By Schur complement, $\Sigma+\Phi+\Pi_{2}+\mathscr{A}^{\mathrm{T}} R \mathscr{A}<0$ is equivalent to (9). This completes the proof.

Remark 6. From (25) and (32), the information $\tau(t) \in$ $\left[h_{1},\left(h_{1}+h_{2}\right) / 2\right]$ and $\tau(t) \in\left[\left(h_{1}+h_{2}\right) / 2, h_{2}\right]$ is considered adequately in our paper, which may lead to less conservative results.

Remark 7. The reciprocally convex optimization technique was utilized in each subinterval $\left[h_{1},\left(h_{1}+h_{2}\right) / 2\right]$ and $\left[\left(h_{1}+\right.\right.$ $\left.\left.h_{2}\right) / 2, h_{2}\right]$, which has potential to yield less conservative conditions.

Remark 8. Based on the results of Theorem 3, an improved stability criterion for system (3) is introduced by utilizing the idea of [17] which divided the bounding of activation function $k_{i}^{-} \leqslant f_{i}\left(z_{i}(t)\right) / z_{i}(t) \leqslant k_{i}^{+}$into two subintervals, such as $k_{i}^{-} \leqslant f_{i}\left(z_{i}(t)\right) / z_{i}(t) \leqslant\left(k_{i}^{-}+k_{i}^{+}\right) / 2$ and $\left(k_{i}^{-}+k_{i}^{+}\right) / 2 \leqslant$ $f_{i}\left(z_{i}(t)\right) / z_{i}(t) \leqslant k_{i}^{+}$. Finally, a numerical example is given to show that Theorem 9 significantly improves the feasible region of stability criterion comparing with some previous literatures.

Theorem 9. For given positive scalars $h_{1}, h_{2}, h=h_{2}-$ $h_{1}$, and any scalars $u_{1}, u_{2}$, diagonal matrices $K_{1}=$ $\operatorname{diag}\left(k_{1}^{-}, k_{2}^{-}, \ldots, k_{n}^{-}\right), K_{2}=\operatorname{diag}\left(k_{1}^{+}, k_{2}^{+}, \ldots, k_{n}^{+}\right)$, and system (3) is globally asymptotically stable for $0 \leqslant h_{1} \leqslant \tau(t) \leqslant h_{2}$, $u_{1} \leqslant \dot{\tau}(t) \leqslant u_{2}$, if there exist symmetric positive matrices $P$, $X=\left[X_{i j}\right]_{2 \times 2}, Q=\left[Q_{i j}\right]_{2 \times 2}, \bar{P}=\left[P_{i j}\right]_{2 \times 2}, R_{i}(i=1,2,3,4)$, positive diagonal matrices $T_{11}, T_{12}, T_{13}, T_{21}, T_{22}, T_{23}, \Delta=$ $\operatorname{diag}\left(\delta_{1}, \delta_{2}, \ldots, \delta_{n}\right), \Lambda=\operatorname{diag}\left(\lambda_{1}, \lambda_{2}, \ldots, \lambda_{n}\right)$, and any matrices $S_{1}, S_{2}$ with appropriate dimensions, satisfying the following LMIs:

$$
\begin{aligned}
& {\left[\begin{array}{cc}
\Sigma+\Phi_{1}+\Pi_{1} & \mathscr{A}^{T} R \\
* & -R
\end{array}\right]<0,} \\
& {\left[\begin{array}{cc}
\Sigma+\Phi_{1}+\Pi_{2} & \mathscr{A}^{T} R \\
* & -R
\end{array}\right]<0,} \\
& {\left[\begin{array}{cc}
\Sigma+\Phi_{2}+\Pi_{1} & \mathscr{A}^{T} R \\
* & -R
\end{array}\right]<0,} \\
& {\left[\begin{array}{cc}
\Sigma+\Phi_{2}+\Pi_{2} & \mathscr{A}^{T} R \\
* & -R
\end{array}\right]<0,}
\end{aligned}
$$




$$
\begin{aligned}
& {\left[\begin{array}{cc}
R_{1} & S_{1} \\
* & R_{1}
\end{array}\right]>0,} \\
& {\left[\begin{array}{cc}
R_{2} & S_{2} \\
* & R_{2}
\end{array}\right]>0,}
\end{aligned}
$$

where

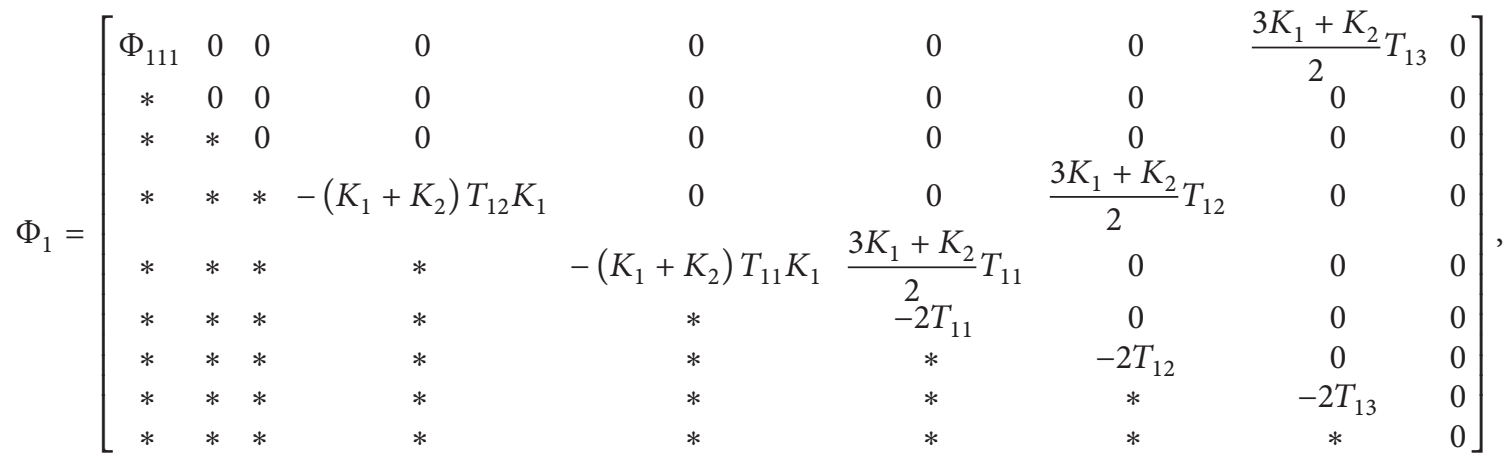

$$
\begin{aligned}
& \Phi_{2}=\left[\begin{array}{ccccccccc}
\Phi_{211} & 0 & 0 & 0 & 0 & 0 & 0 & \frac{K_{1}+3 K_{2}}{2} T_{23} & 0 \\
* & 0 & 0 & 0 & 0 & 0 & 0 & 0 & 0 \\
* & * & 0 & 0 & 0 & 0 & 0 & 0 \\
* & * & * & -\left(K_{1}+K_{2}\right) T_{22} K_{2} & 0 & 0 & \frac{K_{1}+3 K_{2}}{2} T_{22} & 0 & 0 \\
* & * & * & * & -\left(K_{1}+K_{2}\right) T_{21} K_{2} & \frac{K_{1}+3 K_{2}}{2} T_{21} & 0 & 0 & 0 \\
* & * & * & * & * & -2 T_{21} & 0 & 0 & 0 \\
* & * & * & * & * & -2 T_{22} & 0 & 0 \\
* & * & * & * & * & * & * & -2 T_{23} & 0 \\
* & * & * & * & * & * & * & 0
\end{array}\right],
\end{aligned}
$$

where

$$
\Phi_{111}=-\left(K_{1}+K_{2}\right) T_{13} K_{1}, \quad \Phi_{211}=-\left(K_{1}+K_{2}\right) T_{23} K_{2} .
$$

The $\Sigma, \Pi_{1}, \Pi_{2}$ are defined in Theorem 3 .

Proof. Let us choose the same Lyapunov functional proposed in Theorem 3.

Case 1. Consider

$$
k_{i}^{-} \leqslant \frac{f_{i}\left(z_{i}(t)\right)}{z_{i}(t)} \leqslant \frac{k_{i}^{-}+k_{i}^{+}}{2} .
$$

Similar to (19), there exist positive diagonal matrices $T_{11}, T_{12}$, $T_{13}$, such that the following inequalities hold based on (45):

$$
\begin{aligned}
0 \leqslant & -2 f^{T}(z(t)) T_{11} f(z(t)) \\
& +2 z^{T}(t) T_{11}\left(\frac{3 K_{1}+K_{2}}{2}\right) f(z(t)) \\
& -z^{T}(t)\left(K_{1}+K_{2}\right) T_{11} K_{1} z(t) \\
& -2 f^{T}(z(t-\tau(t))) T_{12} f(z(t-\tau(t))) \\
& +2 z^{T}(t-\tau(t)) T_{12}\left(\frac{3 K_{1}+K_{2}}{2}\right) f(z(t-\tau(t)))
\end{aligned}
$$

$$
\begin{aligned}
& -z^{T}(t-\tau(t))\left(K_{1}+K_{2}\right) T_{12} K_{1} z(t-\tau(t)) \\
& -2 f^{T}\left(z\left(t-h_{1}\right)\right) T_{13} f\left(z\left(t-h_{1}\right)\right) \\
& +2 z^{T}\left(t-h_{1}\right) T_{13}\left(\frac{3 K_{1}+K_{2}}{2}\right) f\left(z\left(t-h_{1}\right)\right) \\
& -z^{T}\left(t-h_{1}\right)\left(K_{1}+K_{2}\right) T_{13} K_{1} z\left(t-h_{1}\right) \\
= & \zeta^{T}(t) \Phi_{1} \zeta(t) .
\end{aligned}
$$

Therefore, by the use of proof of Theorem 3, when $k_{i}^{-} \leqslant$ $f_{i}\left(z_{i}(t)\right) / z_{i}(t) \leqslant\left(k_{i}^{-}+k_{i}^{+}\right) / 2, h_{1} \leqslant \tau(t) \leqslant\left(h_{1}+h_{2}\right) / 2$, from (16)-(18), (34), and (46), we obtain

$$
\dot{V}\left(z_{t}\right) \leqslant \zeta^{T}(t)\left(\Sigma+\Phi_{1}+\Pi_{1}+\mathscr{A}^{T} R \mathscr{A}\right) \zeta(t) .
$$

If $\Sigma+\Phi_{1}+\Pi_{1}+\mathscr{A}^{T} R \mathscr{A}<0$, then there exists a scalar $\varepsilon_{2}>0$, such that

$$
\dot{V}\left(z_{t}\right) \leqslant-\varepsilon_{2} \zeta^{T}(t) \zeta(t) \leqslant-\varepsilon_{2} z^{T}(t) z(t)<0, \quad \forall z(t) \neq 0 .
$$

Thus, according to [20], system (3) is globally asymptotically stable for $k_{i}^{-} \leqslant f_{i}\left(z_{i}(t)\right) / z_{i}(t) \leqslant\left(k_{i}^{-}+k_{i}^{+}\right) / 2, h_{1} \leqslant \tau(t) \leqslant$ 
$\left(h_{1}+h_{2}\right) / 2$. By Schur complement, $\Sigma+\Phi_{1}+\Pi_{1}+\mathscr{A}^{T} R \mathscr{A}<0$ is equivalent to (37).

Case 2. Consider

$$
\frac{k_{i}^{-}+k_{i}^{+}}{2} \leqslant \frac{f_{i}\left(z_{i}(t)\right)}{z_{i}(t)} \leqslant k_{i}^{+} .
$$

Similar to (19), there exist positive diagonal matrices $T_{21}, T_{22}$, $T_{23}$, such that the following inequalities hold based on (49):

$$
\begin{aligned}
0 \leqslant & -2 f^{T}(z(t)) T_{21} f(z(t)) \\
& +2 z^{T}(t) T_{21}\left(\frac{3 K_{2}+K_{1}}{2}\right) f(z(t)) \\
& -z^{T}(t)\left(K_{1}+K_{2}\right) T_{21} K_{2} z(t) \\
& -2 f^{T}(z(t-\tau(t))) T_{22} f(z(t-\tau(t))) \\
& +2 z^{T}(t-\tau(t)) T_{22}\left(\frac{3 K_{2}+K_{1}}{2}\right) f(z(t-\tau(t))) \\
& -z^{T}(t-\tau(t))\left(K_{1}+K_{2}\right) T_{22} K_{2} z(t-\tau(t)) \\
& -2 f^{T}\left(z\left(t-h_{1}\right)\right) T_{23} f\left(z\left(t-h_{1}\right)\right) \\
& +2 z^{T}\left(t-h_{1}\right) T_{23}\left(\frac{3 K_{2}+K_{1}}{2}\right) f\left(z\left(t-h_{1}\right)\right) \\
& -z^{T}\left(t-h_{1}\right)\left(K_{1}+K_{2}\right) T_{23} K_{2} z\left(t-h_{1}\right) \\
= & \zeta^{T}(t) \Phi_{2} \zeta(t) .
\end{aligned}
$$

Therefore, by the use of proof of Theorem 3, when $\left(k_{i}^{-}+\right.$ $\left.k_{i}^{+}\right) / 2 \leqslant f_{i}\left(z_{i}(t)\right) / z_{i}(t) \leqslant k_{i}^{+}, h_{1} \leqslant \tau(t) \leqslant\left(h_{1}+h_{2}\right) / 2$, from (16)-(18), (34), and (50), we obtain

$$
\dot{V}\left(z_{t}\right) \leqslant \zeta^{T}(t)\left(\Sigma+\Phi_{2}+\Pi_{1}+\mathscr{A}^{T} R \mathscr{A}\right) \zeta(t) .
$$

If $\Sigma+\Phi_{2}+\Pi_{1}+\mathscr{A}^{T} R \mathscr{A}<0$, then there exists a scalar $\varepsilon_{3}>0$, such that

$$
\dot{V}\left(z_{t}\right) \leqslant-\varepsilon_{3} \zeta^{T}(t) \zeta(t) \leqslant-\varepsilon_{3} z^{T}(t) z(t)<0, \quad \forall z(t) \neq 0 .
$$

Thus, according to [20], system (3) is globally asymptotically stable for $\left(k_{i}^{-}+k_{i}^{+}\right) / 2 \leqslant f_{i}\left(z_{i}(t)\right) / z_{i}(t) \leqslant k_{i}^{+}, h_{1} \leqslant \tau(t) \leqslant$ $\left(h_{1}+h_{2}\right) / 2$. By Schur complement, $\Sigma+\Phi_{2}+\Pi_{1}+\mathscr{A}^{T} R \mathscr{A}<0$ is equivalent to (38).
TABLE 1: Allowable upper bound of $h_{2}$ for different $u_{2}$.

\begin{tabular}{lccc}
\hline Method & $u_{2}=0.1$ & $u_{2}=0.5$ & $u_{2}=0.9$ \\
\hline$[15]$ & 3.65 & 3.32 & 3.26 \\
{$[16]$} & 3.78 & 3.45 & 3.39 \\
{$[17]$} & 4.19 & 3.62 & 3.59 \\
Theorem 3 & 4.97 & 4.01 & 3.90 \\
Theorem 9 & 5.45 & 4.65 & 4.57 \\
\hline
\end{tabular}

\section{Numerical Examples}

In this section, an example is given to demonstrate the effectiveness of the derived method.

Example 1. Consider the stability of neural networks (3) with the following parameters:

$$
\begin{gathered}
C=\left[\begin{array}{cccc}
1.2769 & 0 & 0 & 0 \\
0 & 0.6231 & 0 & 0 \\
0 & 0 & 0.9230 & 0 \\
0 & 0 & 0 & 0.4480
\end{array}\right], \\
A=\left[\begin{array}{cccc}
-0.0373 & 0.4852 & -0.3351 & 0.2336 \\
-1.6033 & 0.5988 & -0.3224 & 1.2352 \\
0.3394 & -0.0860 & -0.3824 & -0.5785 \\
-0.1311 & 0.3253 & -0.9534 & -0.5015
\end{array}\right], \\
B=\left[\begin{array}{cccc}
0.8674 & -1.2405 & -0.5325 & 0.0220 \\
0.0474 & -0.9164 & 0.0360 & 0.9816 \\
1.8495 & 2.6117 & -0.3788 & 0.8428 \\
-2.0413 & 0.5179 & 1.1734 & -0.2775
\end{array}\right], \\
K_{1}=\operatorname{diag}\{0,0,0,0\}, \\
K_{2}=\operatorname{diag}\{0.1137,0.1279,0.7994,0.2368\} \\
f_{1}(s)=0.05685(|s+1|-|s-1|), \\
f_{2}(s)=0.06395(|s+1|-|s-1|), \\
f_{3}(s)=0.3997(|s+1|-|s-1|) \\
f_{4}(s)=0.1184(|s+1|-|s-1|) .
\end{gathered}
$$

For given $h_{1}=3, u_{1}=0$, the upper bounds of $h_{2}$ for different $u_{2}$ are derived by Theorems 3 and 9 and those results in [1517] are listed in Table 1. According to Table 1, our obtained results are much less conservative than those in the literature.

\section{Conclusions}

The problem of delay-dependent asymptotic stability analysis for neural networks with interval time-varying delays is considered based on the delay-partitioning method. Some less conservative stability criteria are established in terms of linear matrix inequalities (LMIs) by constructing a new $\mathrm{L}-\mathrm{K}$ functional in each subinterval and combining with reciprocally convex approach. Finally, a numerical example is given to show the improved stability region of the proposed results. 


\section{Conflict of Interests}

The authors declare that there is no conflict of interests regarding the publication of this paper.

\section{Acknowledgments}

The work is supported by the National Natural Science Foundation of China (Grant no. 71461027) and the doctoral scientific research foundation of Zunyi Normal College (BS[2014]18).

\section{References}

[1] Y. He, M. Wu, and J.-H. She, "Delay-dependent exponential stability of delayed neural networks with time-varying delay," IEEE Transactions on Circuits and Systems II: Express Briefs, vol. 53, no. 7, pp. 553-557, 2006.

[2] Y. He, G. Liu, and D. Rees, "New delay-dependent stability criteria for neural networks with yime-varying delay," IEEE Transactions on Neural Networks, vol. 18, no. 1, pp. 310-314, 2007.

[3] T. Li and X. L. Ye, "Improved stability criteria of neural networks with time-varying delays: an augmented LKF approach," Neurocomputing, vol. 73, no. 4-6, pp. 1038-1047, 2010.

[4] S. Lv, J. Tian, and S. Zhong, "Delay-dependent stability analysis for recurrent neural networks with time-varying delays," Mathematical Problems in Engineering, vol. 2012, Article ID 910140, 14 pages, 2012.

[5] O. M. Kwon and J. H. Park, "Improved delay-dependent stability criterion for neural networks with time-varying delays," Physics Letters, A, vol. 373, no. 5, pp. 529-535, 2009.

[6] S.-P. Xiao and X.-M. Zhang, "New globally asymptotic stability criteria for delayed cellular neural networks," IEEE Transactions on Circuits and Systems II: Express Briefs, vol. 56, no. 8, pp. 659663, 2009.

[7] S. Mou, H. Gao, J. Lam, and W. Qiang, "A new criterion of delaydependent asymptotic stability for Hopfield neural networks with time delay," IEEE Transactions on Neural Networks, vol. 19, no. 3, pp. 532-535, 2008.

[8] H. Zhang, Z. Liu, G.-B. Huang, and Z. Wang, "Novel weightingdelay-based stability criteria for recurrent neural networks with time-varying delay," IEEE Transactions on Neural Networks, vol. 21, no. 1, pp. 91-106, 2010.

[9] L. Hu, H. Gao, and P. Shi, "New stability criteria for CohenGrossberg neural networks with time delays," IET Control Theory and Applications, vol. 3, no. 9, pp. 1275-1282, 2009.

[10] H.-B. Zeng, Y. He, M. Wu, and C.-F. Zhang, "Complete delaydecomposing approach to asymptotic stability for neural networks with time-varying delays," IEEE Transactions on Neural Networks, vol. 22, no. 5, pp. 806-812, 2011.

[11] S. Xu and J. Lam, "A new approach to exponential stability analysis of neural networks with time-varying delays," Neural Networks, vol. 19, no. 1, pp. 76-83, 2006.

[12] S. Mou, H. Gao, W. Qiang, and K. Chen, "New delay-dependent exponential stability for neural networks with time delay," IEEE Transactions on Systems, Man, and Cybernetics, Part B: Cybernetics, vol. 38, no. 2, pp. 571-576, 2008.

[13] Y. Zhang, D. Yue, and E. Tian, "New stability criteria of neural networks with interval time-varying delay: a piecewise delay method," Applied Mathematics and Computation, vol. 208, no. 1, pp. 249-259, 2009.
[14] Y. Wang, C. Yang, and Z. Zuo, "On exponential stability analysis for neural networks with time-varying delays and general activation functions," Communications in Nonlinear Science and Numerical Simulation, vol. 17, no. 3, pp. 1447-1459, 2012.

[15] L. Hu, H. Gao, and W. X. Zheng, "Novel stability of cellular neural networks with interval time-varying delay," Neural Networks, vol. 21, no. 10, pp. 1458-1463, 2008.

[16] T. Li, A. Song, M. Xue, and H. Zhang, "Stability analysis of delayed neural networks based on an improved delaypartitioning approach," Journal of Computational and Applied Mathematics, vol. 235, no. 9, pp. 3086-3095, 2011.

[17] O. M. Kwon, S. M. Lee, J. H. Park, and E. J. Cha, "New approaches on stability criteria for neural networks with interval time-varying delays," Applied Mathematics and Computation, vol. 218, no. 19, pp. 9953-9964, 2012.

[18] S. Boyd, V. Balakrishnan, E. Feron, and L. El Ghaoui, Linear Matrix Inequalities in Systems and Control, SIAM, Philadelphia, $\mathrm{Pa}, \mathrm{USA}, 1994$.

[19] P. Park, J. W. Ko, and C. Jeong, "Reciprocally convex approach to stability of systems with time-varying delays," Automatica, vol. 47, no. 1, pp. 235-238, 2011.

[20] J. Hale, Theory of Functional Differential Equations, Springer, New York, NY, USA, 1977. 


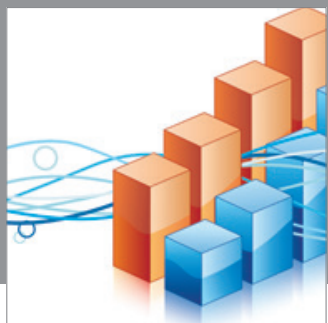

Advances in

Operations Research

mansans

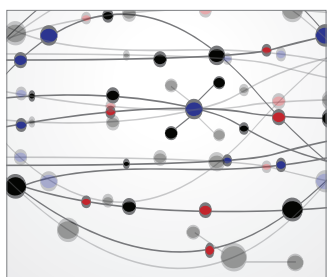

The Scientific World Journal
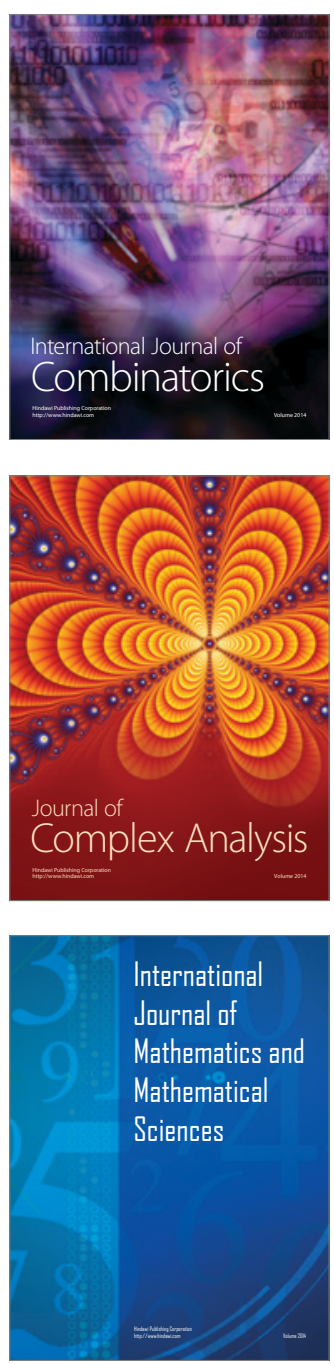
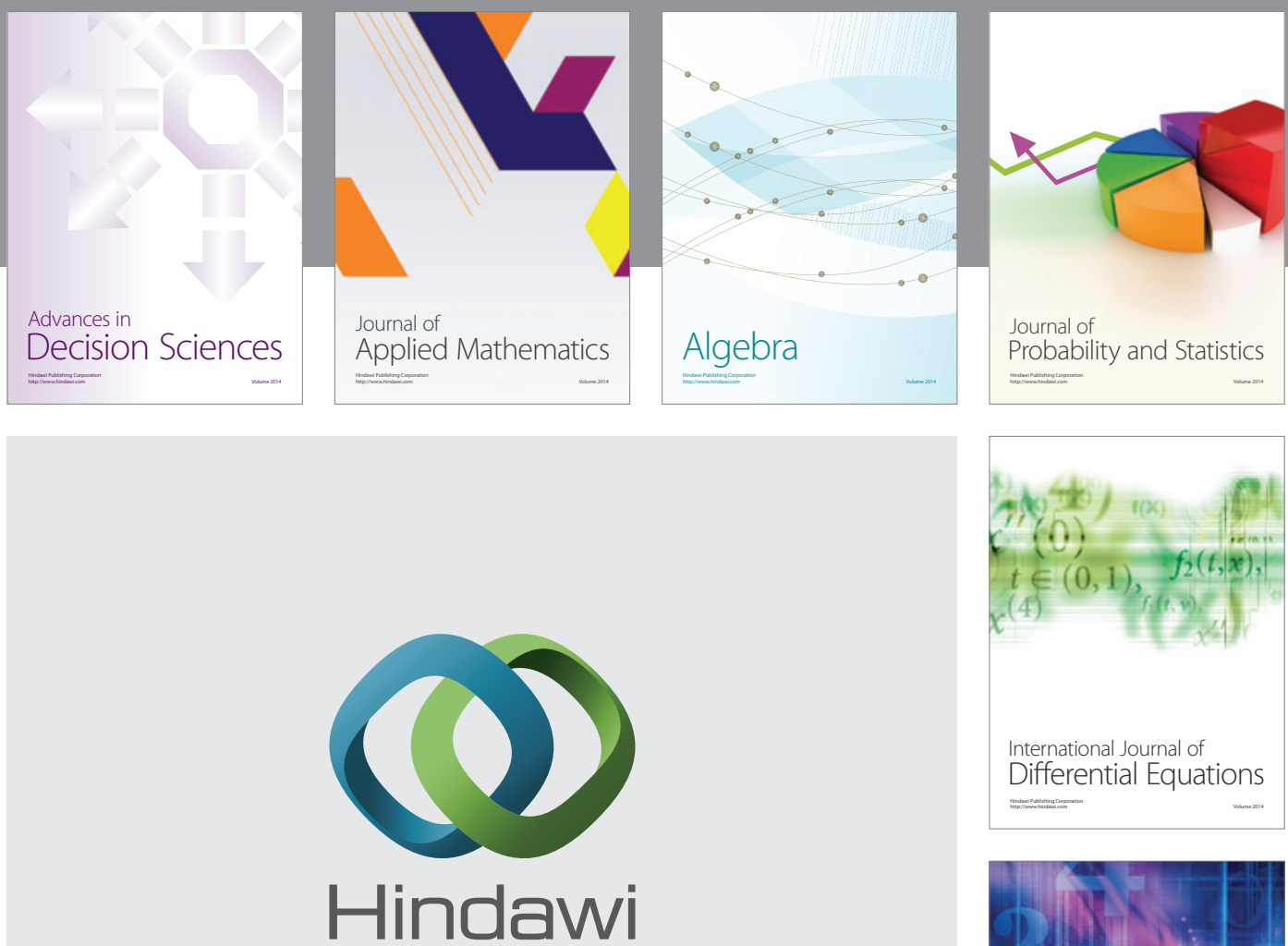

Submit your manuscripts at http://www.hindawi.com
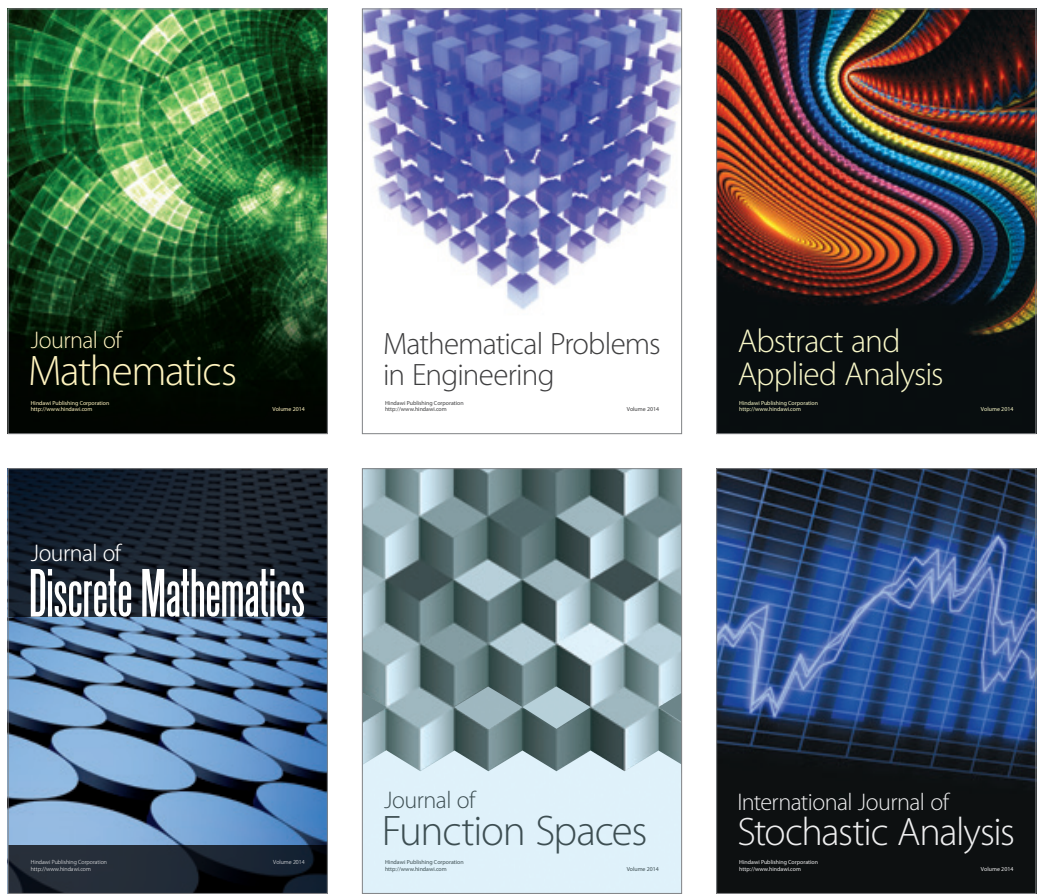

Journal of

Function Spaces

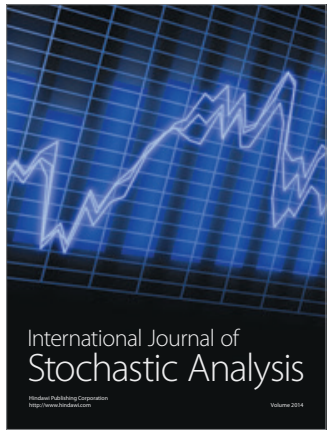

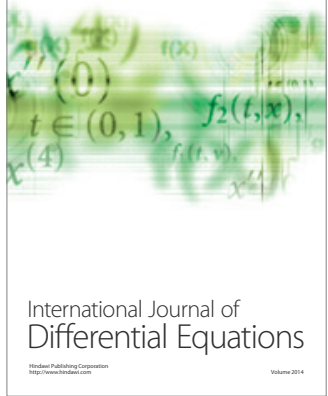
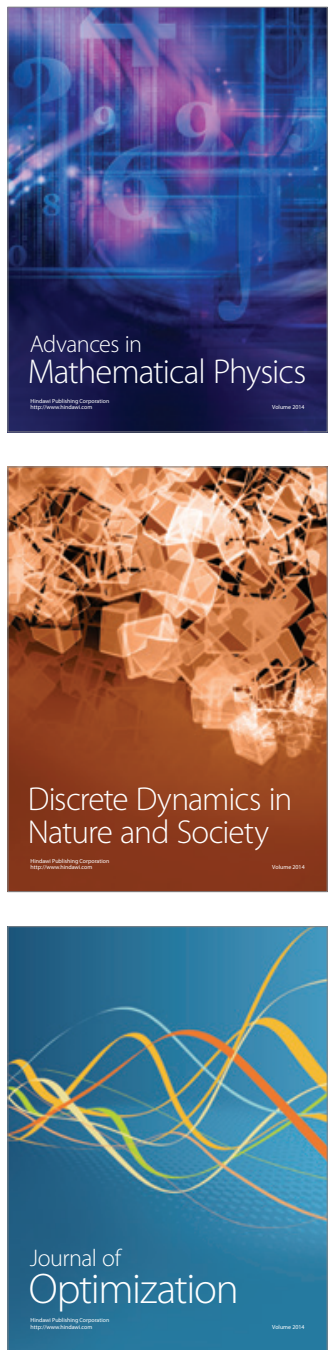